\title{
New Regression models for Estimation daily temperature of Karachi and its Neural Network analysis
}

\author{
Tahir A. ${ }^{1,2 *}$, Ashraf M. ${ }^{1}$, Akhter M. ${ }^{3}$, Uddin Z. ${ }^{2}$ and Sarim M. ${ }^{4}$ \\ 1Department of Physics, Federal Urdu University of Arts Science and Technology, Karachi, Pakistan \\ 2Department of Physics, University of Karachi, Karachi, Pakistan \\ ${ }^{3}$ Department of Applied Physics, University of Karachi, Karachi, Pakistan \\ ${ }^{4}$ Department of Computer Science, Federal Urdu University of Arts Science and Technology, Karachi, Pakistan \\ Received: 11/09/2021, Accepted: 29/10/2021, Available online: 30/10/2021 \\ *to whom all correspondence should be addressed: e-mail: adeel.tahir@fuuast.edu.pk
} https://doi.org/10.30955/gnj.003953

\section{Graphical abstract}

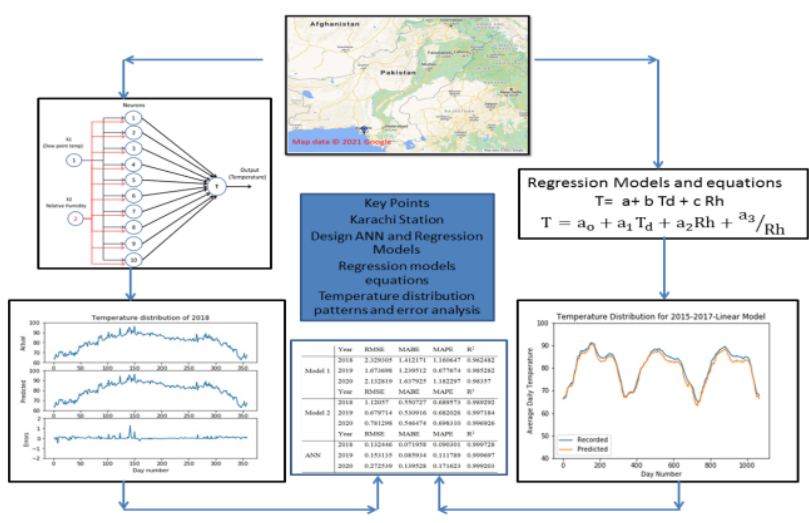

\section{Abstract}

This study presents the determination of the average daily temperature distribution for Karachi city. Artificial Neural Network (ANN) has been used to predict the average daily temperature of 2018, 2019, and 2020. Two regression models (linear and non-linear) were also developed. These models are based on relative humidity and dew points. Karachi's six-year environmental datasets were used for the case study location and to establish temperature distribution models. In ANN three years, temperature data (2015-2017) was used to train and validate the ANN model. The same data was used to find the regression coefficients of each model. Both models and ANN are then used to estimate the average daily temperature of years 2018-2020. The statistical errors are also calculated for comparison and to evaluate the performance of both models; an excellent agreement was found between recorded and ANN estimates. Both regression models predict average daily temperature with reasonable uncertainties. However, the non-linear regression model predictions are better. The results show that the models provide a good prediction of temperature distribution.

Keywords: Neural network, regression, Karachi, mathematical modeling, temperature distribution.

\section{Introduction}

Climate changes influence the environment and play an essential role in daily routine life. The change can be predicted by gathering information, observing, and forecasting weather events over the years (Ukhurebor et al., 2017). The climatic change depends on solar radiation flux, cloudiness, longitude, and latitude (McHugh et al., 2015). Temperature is an important parameter that affects different weather elements such as dew point temperature, precipitation, humidity, clouds, and atmospheric pressure (Pondyal et al., 2011). The moisture in the air can easily be measured by relative humidity and dew point temperature (Iqbal, 2011). The performance of metals, processing food, biological items, and electrical devices are also affected by relative humidity (Lawrence, 2005). Solar radiation, ambient temperature, and dew point are the factors whose combination influences relative humidity (Geerts, 2003). For human comfort, thunderstorms probability, rain, fog, and frost forecasting, Meteorologists adopted dew point temperature irrespective of relative humidity (Eludoyin et al., 2014; Tanabe, et al., 1994). At lower atmosphere pressure, the dew point greater than $60^{\circ} \mathrm{C}$ shows intense thunderstorm probability (Górnicki et al., 2017). When relative humidity is less than $10 \%$ and air temperature ranges from 50 to $60^{\circ} \mathrm{F}$, the negative dew point is observed in a semiarid environment (Yousif et al., 2013). At the same time, 13 to $20^{\circ} \mathrm{F}$ dew point temperature is critical and leads to cold nights with possible difficulty in keeping room temperatures above critical levels. At dew point below zero, the moisture content in the air is less. Besides precipitation, dewfall and direct water vapor adsorption are the main mechanisms that add water to the soil (Ukhurebor et al., 2017). It is believed that world advancement has made an ordinary person's life more comfortable, but it is a fact that this advancement has caused global temperature to rise. The estimated global warming trend is $2^{\circ} \mathrm{C}$ per century (Salinger, 2005). In the last century, in climatic variation research, the focus was the variation of surface air temperature. In Pakistan, the 
air temperature has been studied since 1882 (Pondyal et al., 2011). It is found that Normal distribution fits well on temperature distribution and a temperature increase from $0.3^{\circ} \mathrm{C}$ to $1.0^{\circ} \mathrm{C}$ in the twentieth century. Bayesian analysis computational technique shows that the average annual mean temperature for $1882-1960$ is less than the period 1961-2000 (Agam et al., 2006).

Monthly, seasonal, and annual observations were used to study the pattern of maximum and minimum temperatures of 39 weather stations in Pakistan. Two statistical tests (Sen's slope and Mann-Kendall) were used to classify the slopes and magnitude of the climate change trend. The statistical study examined the potential variations in the maximum and minimum temperature patterns. The maximum and minimum temperatures for 2030 and 2060 is forecast using a statistical downscaling climate prediction model (SimCLIM). With the median Representative Concentration Pathway (RCP-6.0) for potential predictions in SimCLIM, an ensemble of 40 General Circulation Models (GCMs) was used. In February and March, a positive trend from 0.06 to $0.51^{\circ} \mathrm{C}$, whereas in Balochistan and northern areas of Pakistan negative trend from -0.06 to $-0.30^{\circ} \mathrm{C}$ temperature has been observed (Amin et al., 2018). Variation in Karachi temperature was studied from 1947 to 2005 (59 years) by evaluating time series data of mean annual maximum, mean annual minimum, and mean annual temperature. Taking anomalies into account and using linear regression analysis, an increase of around $4-6^{\circ} \mathrm{C}$ was suggested (Sajjad et al., 2009). Temperature rise raises the saturation pressure exponentially and impacts the air strength of water vapor retaining (Gong et al., 2006). Moisture in the air can be estimated by relative humidity $\mathrm{R}_{\mathrm{H}}$ and dew point temperature $\mathrm{T}_{\mathrm{d}}$. A simple thumb rule for approximating moist air conversion is that if $\mathrm{R}_{H}>50 \%$, a decrease of $5 \%$ in $R_{H}$ causes $1^{\circ} \mathrm{C}$ decrease in $T_{d}$ (Lawrence, 2005). Relative humidity $\left(R_{H}\right)$ is the ratio between the amount of water the ambient air holds and the amount it could have when the air is saturated with water vapor at the same temperature. Therefore, it can be expressed as the ratio between the actual vapor pressure $\left(e_{a}\right)$ and saturation $\left(e_{s}\right)$. The saturation vapor pressure from the ambient temperature and the real vapor pressure from the dew point temperature $\left(T_{d}\right)$ can be calculated using empirical formulas.

$$
e(T)=0.6108 \exp \left(\frac{17.62 T}{T+273.3}\right)
$$

An empirical model to estimate dew point $\left(T_{d}\right)$ by parameters daily minimum $\left(T_{\mathrm{min}}\right)$ and daily maximum $\left(T_{\max }\right)$ temperature and the estimated daily potential evapotranspiration ratio to annual precipitation $\left(E F=I_{E P}\right.$, day $/ I_{p}$, ann) by Kimball.

$$
\begin{aligned}
& T_{d}=T_{\min }\left\{-0.127+1.121\left(1.003-1.444 E F+12.312 E F^{2}\right.\right. \\
& \left.\quad-32.766 E F^{3}\right)+\left(0.00006\left(T_{\max }-T_{\min }\right)\right\}
\end{aligned}
$$

Another model developed by Sboarina and Cescatti (2004) to measure dewpoint temperature (Td) using daily minimum ( $\left.T_{\min }\right)$ and daily maximum $\left(T_{\max }\right)$ and daily mean ( $\left.T_{\text {mean }}\right)$ temperature.

$$
T_{d}=T_{\text {min }}-k\left(0.45 T_{\text {max }}-0.55 T_{\text {mean }}-T_{\text {min }}\right)
$$

$\mathrm{k}$ is a site-specific constant (Al-Muhyi et al., 2016; Gunawardhana et al., 2017).

\section{Study area}

Karachi is Pakistan's first, the world's twelfth largest city and capital of Sindh province situated at geographical location (latitude) $24^{\circ} 51^{\prime} 39.4776^{\prime \prime} \mathrm{N}$ and (longitude) $66^{\circ}$ 59' 25.8036". It is located south coast of Pakistan and covers an area of around $3,527 \mathrm{~km}^{2}$ (Figure 1).

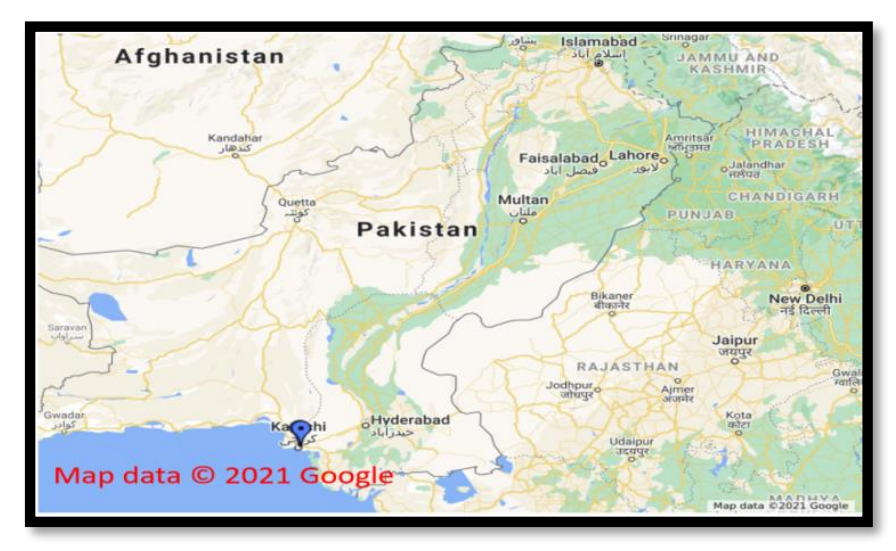

Figure 1. Geographical location of Karachi, Pakistan (Google Maps. Retrieved Jan 2021). (Google Maps. Retrieved Jan 2021, from https://www.google.com/maps/ @24.920064,67.1055872,11z).

Karachi is Pakistan's most cosmopolitan region, with a linguistic, ethnic, and religious diversity that makes it one of the world's most secular and socially liberal cities. Karachi serves as a transportation center due to its location in the Arabian Sea. It hosts Pakistan's two largest seaports, the Port of Karachi and Port Bin Qasim, and its busiest airport, Jinnah International Airport (Karachi - The dominant Culture. Retrieved December 2020, from History Pak,com: https://historypak.com/karachisdominant-culture).

Karachi has an arid climate with a long "Summer Season" moderated by the Arabian Sea's oceanic impact. May and June are the hottest months where the temperature reaches around $43^{\circ} \mathrm{C}$. Spring and Autumn in Karachi have a short period. The monsoon rainfall receives from July to September. Almost 7 inches per annum is average rainfall. Winters are moderate, with temperatures as low as $5^{\circ} \mathrm{C}$ in January. Karachi has a tropical climate with hot summers and mild winters. Due to its geographical location, Karachi is the most important city of Pakistan and neighboring countries.

\section{Methodology}

\subsection{Artificial neural network}

The prediction of average daily temperature, like other meteorological is important for different sectors, an 
industry of renewable energy, agriculture, and everyday life of a common man. Water contents in the soil also depend on temperature, which directly affects processes like seed germination, plant growth, etc. In designing houses, hospitals, etc., the variation in daily temperature is also considered. Scientists and researchers in forecasting temperature have employed various methods. Artificial Neural Network is one of them. In Artificial Table 1. Weights of the input variable, and neurons and bias associated with the neurons

\begin{tabular}{|c|c|c|c|c|}
\hline $\mathbf{I}$ & $W_{1 i}$ & $\mathbf{W}_{2 i}$ & $\mathbf{w}_{\mathbf{i}}$ & $b_{i}$ \\
\hline 1 & 4.060232 & 5.971542 & 0.021858 & -8.46554 \\
\hline 2 & -1.59832 & -10.87 & 0.003273 & 3.03792 \\
\hline 4 & 7.620068 & 0.648445 & 0.001072 & -2.4725 \\
\hline 5 & 1.652472 & 3.545221 & 0.00681 & -0.42737 \\
\hline 6 & -0.22734 & 1.347246 & 0.716058 & -0.54587 \\
\hline 7 & 3.392446 & 5.748316 & 0.002744 & 1.983417 \\
\hline 8 & 0.584383 & -0.59251 & 3.050216 & -0.21358 \\
\hline 9 & -0.26117 & 3.264822 & -0.8349 & 3.474654 \\
\hline 10 & -2.25312 & 3.579778 & -0.79087 & -6.10823 \\
\hline
\end{tabular}

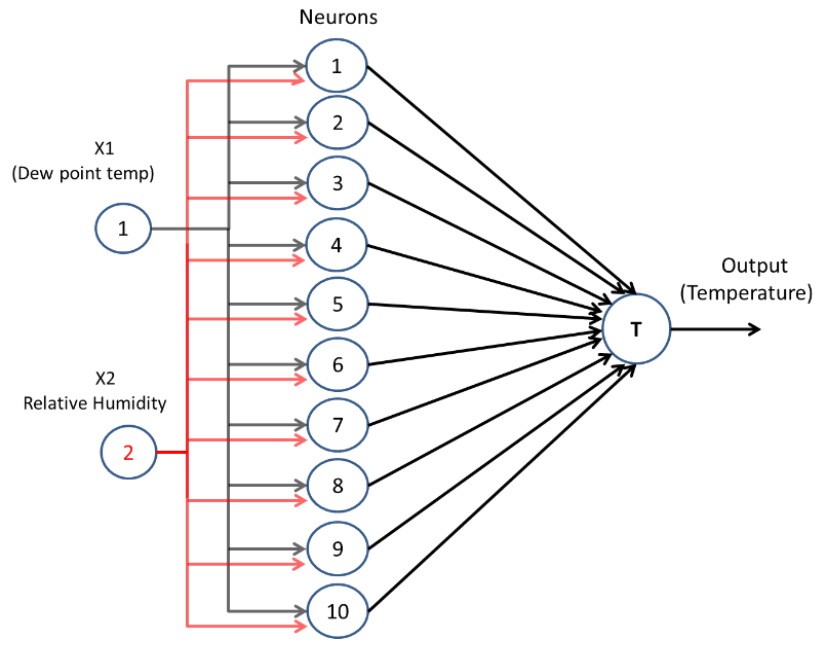

Figure 2. Feedforward neural network with 10 Neuron.

There are two inputs which are dew point and relative humidity, to estimate the temperature. This prediction model is developed on MATLAB, which is a three-layered feed-forward network. The input layer contains two neurons without any hidden layer, 10 neurons are included in the hidden layers. Hidden layer neurons have sigmoidal transfer function while neuron in output layer has linear transfer function. The output of the only neuron in the output layer represents Estimated temperature T, and it is given by

$$
T=\sum_{i=1}^{10} w_{i} H_{i}+B
$$

Where bias $\mathrm{B}=0.47363$ and weights $\mathrm{w}_{\mathrm{i}}$ are given in Table 1 . Here, $\mathrm{H}_{\mathrm{i}}$ can be calculated by the following equation

$$
H_{i}=\frac{1}{1+e^{-E_{i}}}
$$

$E_{i}$ can be calculated using the following formula,
Neural Network, the computer learns the behavior of the input data and performs several tasks to train a machine. Once the machine is trained efficiently, the output data is predicted (Agatonovic, 2000; Malone, 1955; Maqsood et al., 2004). In this study, ANN is used to predict the average daily temperature of Karachi city. The architecture of ANN used in this study is shown Figure 2. 
Model 2: (Non-linear model)

$$
T=\mathrm{a}_{\mathrm{o}}+\mathrm{a}_{1} \mathrm{~T}_{\mathrm{d}}+\mathrm{a}_{2} R h+\frac{\mathrm{a}_{3}}{\mathrm{Rh}}
$$

The Adaptive Neuro-Fuzzy Inference System (ANFIS) and Artificial Neural Networks (ANNs) models were designed to evaluate the monthly air temperature based on latitude, longitude, and altitude at 30 different weather stations of Iran. The monthly model data of 20 and 10 weather stations were used for training and testing, respectively (Kisi et al., 2014). Hietaharju presented a dynamic model for the prediction of indoor temperature in a building. Their model used the precursor method; the precursors were indoor and outdoor temperatures earlier (Hietaharju et al., 2018). Alvares et al., monthly mean air temperature for Brazil used latitude, longitude, and height of the site as independent variables in their regression model (Alvares et al., 2013). In modeling temperature, Wang et al., considered strong seasonality of the temperature distribution and used the sine function of time in days in their model (Wang et al., 2015).

\section{Statistical errors for validation of regression analysis}

The Mean Square Error (MSE), Mean Absolute Error (MABE), Mean Absolute Percent Error (MAPE), and Rsquare are calculated to check the validity of regression analysis results.

$$
\begin{aligned}
& M S E=\frac{1}{n} \sum_{i=1}^{n}\left(T_{c . i}-T_{m . i}\right)^{2} \\
& M A B E=\frac{1}{n} \sum_{i=1}^{n}\left|T_{c . i}-T_{m . i}\right| \\
& M A P E=\frac{1}{n} \sum_{i=1}^{n}\left|\frac{\left(T_{c . i}-T_{m . i}\right)}{T_{m . i}}\right| \times 100 \\
& R^{2}=1-\frac{\sum_{i=1}^{n}\left(T_{c . i}-T_{m . i}\right)^{2}}{\sum_{i=1}^{n}\left(T_{c . i}-\overline{T_{m}}\right)^{2}}
\end{aligned}
$$

\section{Result and discussion}

In this study, ANN is used to estimate the average daily temperature. Neural Network consists of two input variables (dew point and relative humidity), a hidden layer with 10 neurons, and the output variable (Average daily temperature). The network is trained using 1092 data (2015-2017) points (average daily temperature, dew point, and relative humidity) using Levenberg-Marquardt algorithm (Kişi, 2007). 764 out of these 1092 samples were used to train this network, while 164 were used for validation and testing. We used backpropagation algorithm and Levenberg Marquardt gives faster conversion for this algorithm. Later this trained network was used to estimate the 1086 values of temperatures of 2018 to 2020. Predicted and actual values along with errors are plotted in Figure. 3. The Weights $W_{1}$ and $W_{2}$ of the neuron that connects different nodes are determined and presented in Table 1. Table also gives the bias values in equation (3). The bias ' $B$ ' in equation 1 is found to be
0.47363 . The weights $w_{i}$ at nodes connecting neurons in the hidden layer and out are also given in Table 1 . The seasonal variations were removed using moving average method by taking running averages of 25 values.
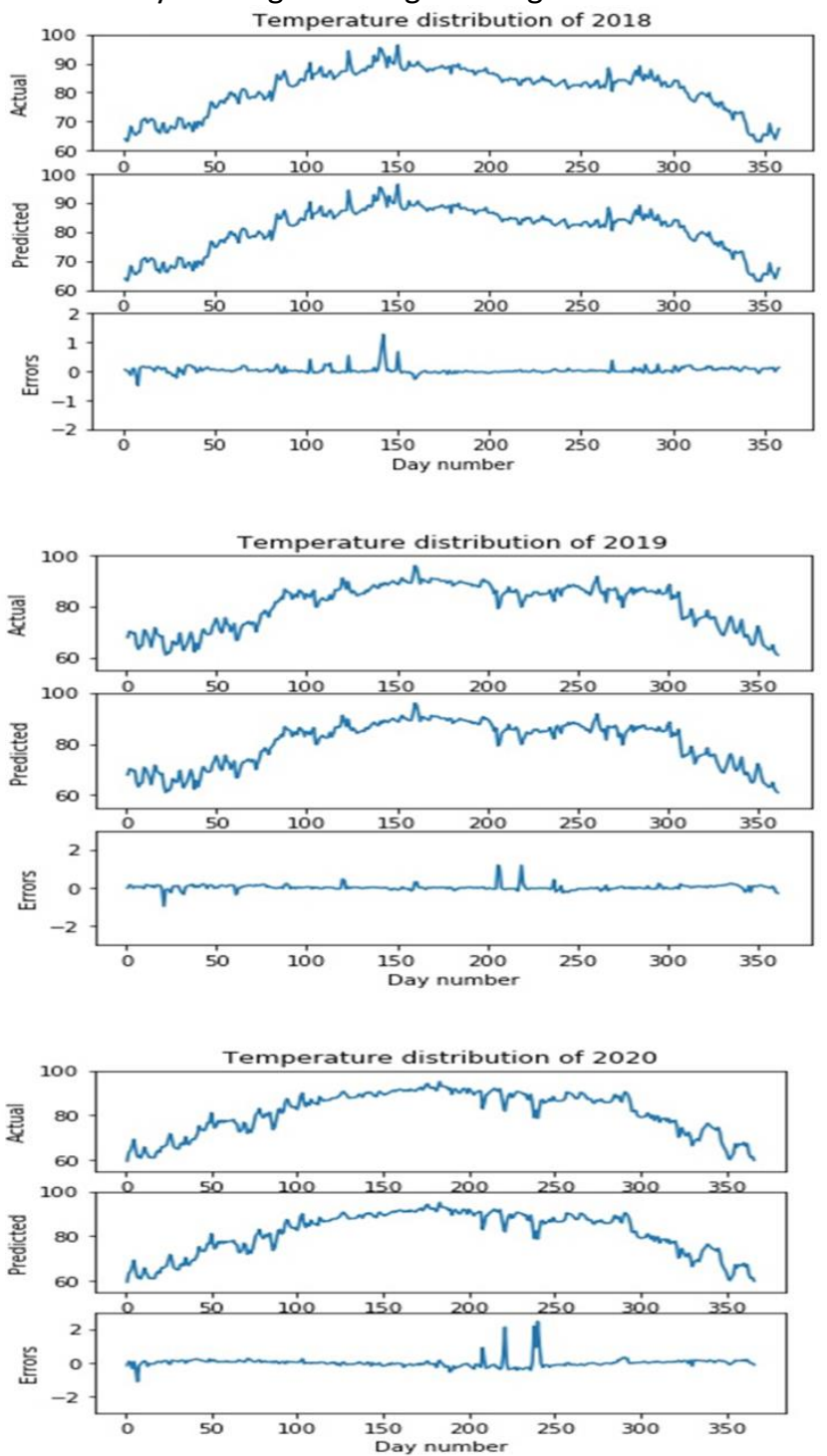

Figure 3 Comparison of predicted and actual average daily temperature of Karachi for the years 2018-2020.

Two multiple regression models are suggested for the average daily temperature distribution. Six years of environmental data is used to establish and validate the two models. The first part of the dataset is also used to find the regression coefficients. These coefficients are substituted in equations 4 and 5 (see equations 10 and 11). For further validation, statistical errors are calculated, and the performance of the models is compared. These errors include Mean Square

Error (MSE), Mean Absolute Error (MABE), Mean Absolute Percent Error (MAPE), and R- square. The best model was identified by predicting temperature distribution for 20182020 and comparing the associated errors with the models.

$$
T=50.02922+1.043707 \mathrm{~T}_{\mathrm{d}}-0.63266 R h
$$




$$
T=34.72128+1.076365 \mathrm{~T}_{\mathrm{d}}-0.48742 \mathrm{Rh}+302.5679 / \mathrm{Rh}
$$

Figure 4 Shows measured and predicted temperature distributions for 2015-2017 by (a) model 1 and (b) model 2. The regression coefficients are determined by multiple regression analysis, and these coefficients are then employed to predict temperature distribution for 20182020 (see Figures 5-7). Similarly, model 2 was used to find the regression coefficients from the temperature distribution of 2015-2017 (see Figure 4). The coefficients are employed to estimate temperature distribution for 2018-2020 (see Figures 5-7).

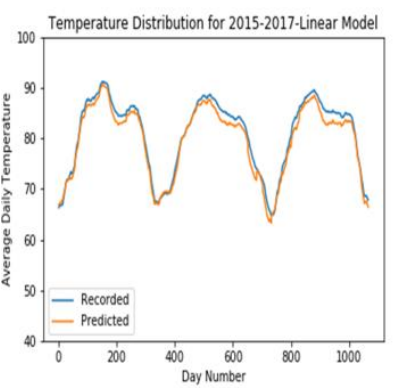

(a)

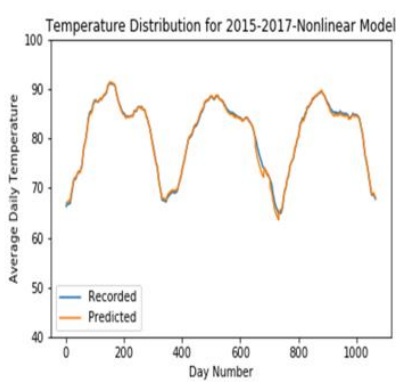

(b)
Figure 4. The recorded and predicted temperature distribution (by (a) model 1 and (b) model 2) for the years 2015-2017.

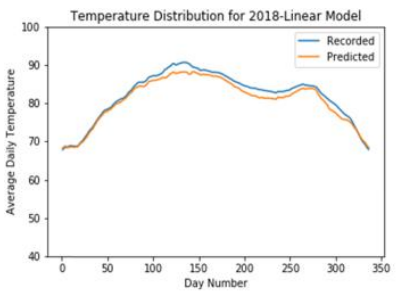

(a)

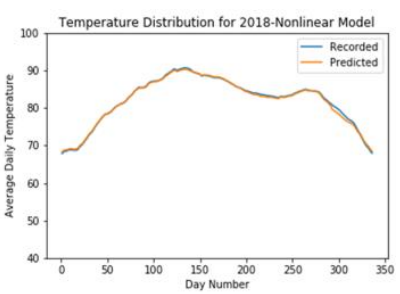

(b)
Figure 5. The recorded and predicted temperature distribution (by (a) model 1 and (b) model 2) for 2018.

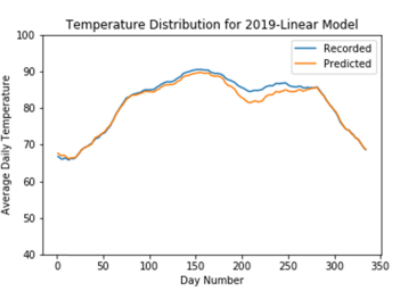

(a)

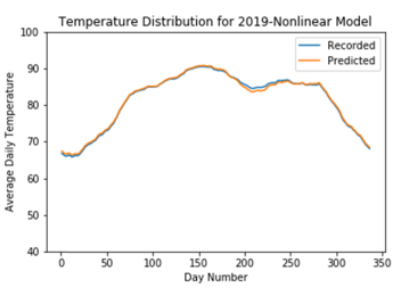

(b)
Figure 6. The recorded and predicted temperature distribution (by (a) model 1 and (b) model 2) for 2019.

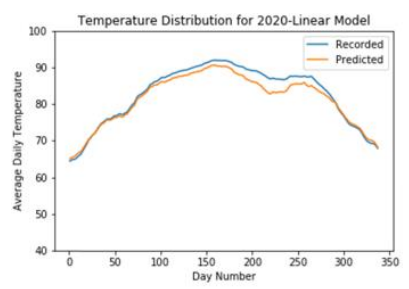

(a)

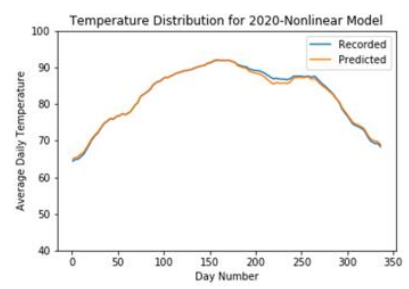

(b)
Figure 7. The recorded and predicted temperature distribution (by (a) model 1 and (b) model 2) for 2020.

Table 2 shows statistical errors in ANN and both regression models. The statistical errors RMSE, MABE, and MAPE, are the lowest in ANN. The coefficient of determination between predicted and actual average daily temperatures is highest for ANN. The maximum absolute difference between measured and calculated temperature by model 1 was greater than $6.23^{\circ} \mathrm{F}$ in 2019 and $7.80^{\circ} \mathrm{F}$ for the year 2020 . Whereas the corresponding difference for model 2 was found to be $2.30^{\circ} \mathrm{F}$ for 2019 and $2.04^{\circ} \mathrm{F}$ for 2020 . The statistical errors correspond to models are listed in table 2 . The table clearly shows that all four statistical errors for model 2 are less than those for model 1. Both temperature distribution and statistical error predictions indicate that model 2 is better than model 1 , and it predicts more reliable temperature values for Karachi city.

The persistence models work well when weather patterns change very little, and features on the weather maps move very slowly. It shows the best result for short-term forecasting ( 2 to 4 days) or where the weather parameters slightly change; otherwise, it's invalid. Accuracy of weather forecasting is better for a short period as compared to an extended period. According to the National Oceanic and Atmospheric Administration (NOAA), around $80 \%$ accurate forecast is observed for the next 5 days, whereas it becomes $50 \%$ for the next 10 days. Furthermore, precise forecasting depends on the geographical location and atmosphere since the weather is non-linear and complex. Some mathematical models are designed to estimate the weather parameters whose accuracy can be justified by statistical error values (Persistence Method. Retrieved November 2020, from http://ww2010.atmos.uiuc.edu/(Gh)/guides/mtr/fcst/mth /prst.rxml).

Table 2. Statistical Errors in the predicted temperature distribution of 2018-2020 for model 1, model 2, and ANN

\begin{tabular}{|c|c|c|c|c|c|}
\hline & Year & RMSE & MABE & MAPE & $\mathbf{R}^{2}$ \\
\hline & 2018 & 2.329305 & 1.412171 & 1.160647 & 0.962482 \\
\hline \multirow[t]{4}{*}{ Model 1} & 2019 & 1.673698 & 1.239512 & 0.677674 & 0.985282 \\
\hline & 2020 & 2.132819 & 1.637925 & 1.182297 & 0.98357 \\
\hline & Year & RMSE & MABE & MAPE & $\mathrm{R}^{2}$ \\
\hline & 2018 & 1.12057 & 0.550727 & 0.689573 & 0.989292 \\
\hline \multirow[t]{4}{*}{ Model 2} & 2019 & 0.679714 & 0.530916 & 0.682026 & 0.997184 \\
\hline & 2020 & 0.781298 & 0.546474 & 0.698310 & 0.996926 \\
\hline & Year & RMSE & MABE & MAPE & $R^{2}$ \\
\hline & 2018 & 0.132446 & 0.071958 & 0.090301 & 0.999728 \\
\hline \multirow[t]{2}{*}{ ANN } & 2019 & 0.153135 & 0.085934 & 0.111789 & 0.999697 \\
\hline & 2020 & 0.272539 & 0.139528 & 0.171623 & 0.999203 \\
\hline
\end{tabular}




\section{Conclusion}

In this study, we modeled the Average daily temperature distribution for Karachi, Pakistan. The study was divided into two parts. In the first part, we used ANN, in which we trained the machine on temperature variations for the years 2015-2017. The input variables were dew point and relative humidity; ANN had one hidden layer consists of 10 neurons. The output was an average daily temperature. The weights and biases obtained after training were used to predict the average daily temperature for three years (2018 to 2020). The predictions by ANN were excellent. The errors are \pm 1 for the years 2018, 2019, whereas the errors for 2020 are in the range \pm 2 . The Average daily temperature can be found through ANN with two independent variables, dew point, and relative humidity. This fact is used in the development of two regression models, one of them is linear (model 1 ), and the other is a non-linear (model 2 ) multiple regression model.

In both models, the regression coefficients were calculated from daily average temperature data of 20152017. These coefficients are then employed in models 1 and 2, and predicted temperatures for 2018-2020. To check the validity of ANN and regression models, statistical errors RMSE, MABE, and MAPE are calculated. The coefficient of determination is also estimated to study the correlation between predicted and actual temperatures. The RMSE, MABE, and MAPE are lowest in ANN (between 0 and 0.3 ), whereas the same errors have the highest model 1 (between 0 and 3). These errors for model 2 are less than 1, except for 2018, i.e., 1.12057. It is also clear from the Figures 5-7 that the overlapping of graphs of actual and predicted temperatures is suitable for model 2 compared to model 1 . We conclude that ANN indicates the best results out of three studies and suggests that model 2 can predict Karachi's daily average temperature.

\section{Acknowledgements}

We are thankful to Meteorological Department of Pakistan, Karachi for providing the data for this study. The data is also available on the web site https://en.tutiempo.net/climate/ws417800.html.

\section{References}

Abed F.M. (2018). Design and fabrication of a multistage solar still with three focal concentric collectors. Journal of Solar Energy Engineering, 140(4).

Agam N. (2006). Dew formation and water vapor adsorption in semi-arid environments-A review. Journal of Arid Environments, 65(4), 572-590.

Agatonovic-Kustrin S. (2000). Basic concepts of artificial neural network (ANN) modeling and its application in pharmaceutical research. Journal of Pharmaceutical and Biomedical Analysis, 22(5), 717-727.

Al-Muhyi A.H.A., Bashar A.L.A.J. and Kwyes L.D.A. (2016). A the study of climate change using statistical analysis case study temperature variation in basra. International Journal of Academic Research, 3, 2(5).
Alvares C.A. (2013). Modeling monthly mean air temperature for Brazil. Theoretical and Applied Climatology, 113(3), 407-427.

Amin A.N. (2018). Evaluation and analysis of temperature for historical (1996-2015) and projected (2030-2060) climates in Pakistan using SimCLIM climate model: ensemble application. Atmospheric Research, 213, 422-436.

Chabane F.M. (2018). MoummiForecast of relationship between a relative humidity and a dew point temperature. Journal of Power Technologies, 98(2), , 183-187.

Eludoyin O.M. (2014). Air temperature, relative humidity, climate regionalization and thermal comfort of Nigeria. International Journal of Climatology, 34(6), 2000-2018.

Geerts B. (2003). Empirical estimation of the monthly-mean daily temperature range. Theoretical and Applied Climatology, 74(3), 145-165.

Gong L.X. (2006). Sensitivity of the Penman-Monteith reference evapotranspirationto key climatic variables in the Changjiang (Yangtze River) basin. Journal of Hydrology, 329(3), 620-629.

Google Maps. (n.d.). Retrieved Jan 2021, from https://www. google.com/maps/@24.920064,67.1055872,11z

Górnicki K.W. (2017). Evaluation of models for the dew point temperature determination. Technical Sciences/University of Warmia and Mazury in Olsztyn, pp. 241-257.

Gunawardhana L.N. (2017). An alternative method for predicting relative humidity for climate change studies. Meteorological Applications, 24(4), 551-559.

Hietaharju P.R. (2018). A dynamic model for indoor temperature prediction in buildings. Energies, 11(6), 1477.

How Temperature and Humidity are Related. (n.d.). Retrieved December 2020, from Science: https://sciencing.com/ temperature-ampamp-humidity-related-7245642.html

Iqbal M.J. (2011). Measuring temperature variability of five major cities of Pakistan. Arabian Journal of Geosciences, 4(3-4), 595-606.

Karachi - The dominant Culture. (n.d.). Retrieved December 2020, from History Pak,com: https://historypak.com/ karachis-dominant-culture/

Kisi O. (2014). Prediction of long-term monthly air temperature using geographical inputs. International Journal of Climatology, 34(1), 179-186.

Kişi Ö. (2007). Streamflow forecasting using different artificial neural network algorithms. Journal of Hydrologic Engineering, 12(5), 532-539.

Lawrence M.G. (2005). The relationship between relative humidity and the dewpoint temperature in moist air: A simple conversion and applications. Bulletin of the American Meteorological Society, 86(2), 225-234.

Malone T.F. (1955). Application of statistical methods in weather prediction. Proceedings of the National Academy of Sciences of the United States of America, 41(11), 806.

Maqsood I.K. (2004). An ensemble of neural networks for weather forecasting. Neural Computing \& Applications, 13(2), 112-122.

McHugh T.A. (2015). Water from air: an overlooked source of moisture in arid and semiarid regions. Scientific Reports, 5(1), 1-6.

Persistence Method. (n.d.). Retrieved November 2020, from http://ww2010.atmos.uiuc.edu/(Gh)/guides/mtr/fcst/mth/p rst.rxml 
Pondyal K.N. (2011). Solar radiation potential at four sites of Nepal. Journal of the Institute of Engineering, 8(3), 189-197.

Sajjad S.H., Hussain B., Khan M.A. and Raza A.Za. (2009). On rising temperature trends of Karachi in Pakistan. Climatic Change, 96(4), 539-547.

Salinger M.J. (2005). Increasing climate variability and change: reducing the vulnerability. In Increasing Climate Variability and Change. Springer, Dordrecht. pp. 1-3.

Tanabe S. (1994). Effects of air temperature, humidity, and air movement on thermal comfort under hot and humid conditions. American Society of Heating, Refrigerating and Air-Conditioning Engineers, Inc., Atlanta, 100(2), 953-969.

Ukhurebor K.E. (2017). Relationship between relative humidity and the dew point temperature in Benin City, Nigeria. Journal of Applied Sciences and Environmental Management, 21(5), 953-956.

Ukhurebor K.E. (2017). The influence of air temperature on the dew point temperature in Benin City, Nigeria. Journal of Applied Sciences and Environmental Management, 21(4), 657-660.

Wang Z.L. (2015). Modeling and forecasting average temperature for weather derivative pricing. Advances in Meteorology.

Yousif T.A. (2013). The relationship between relative humidity and the dew point temperature in Khartoum State, Sudan. Journal of Applied and Industrial Sciences, 1(5), 20-23. 